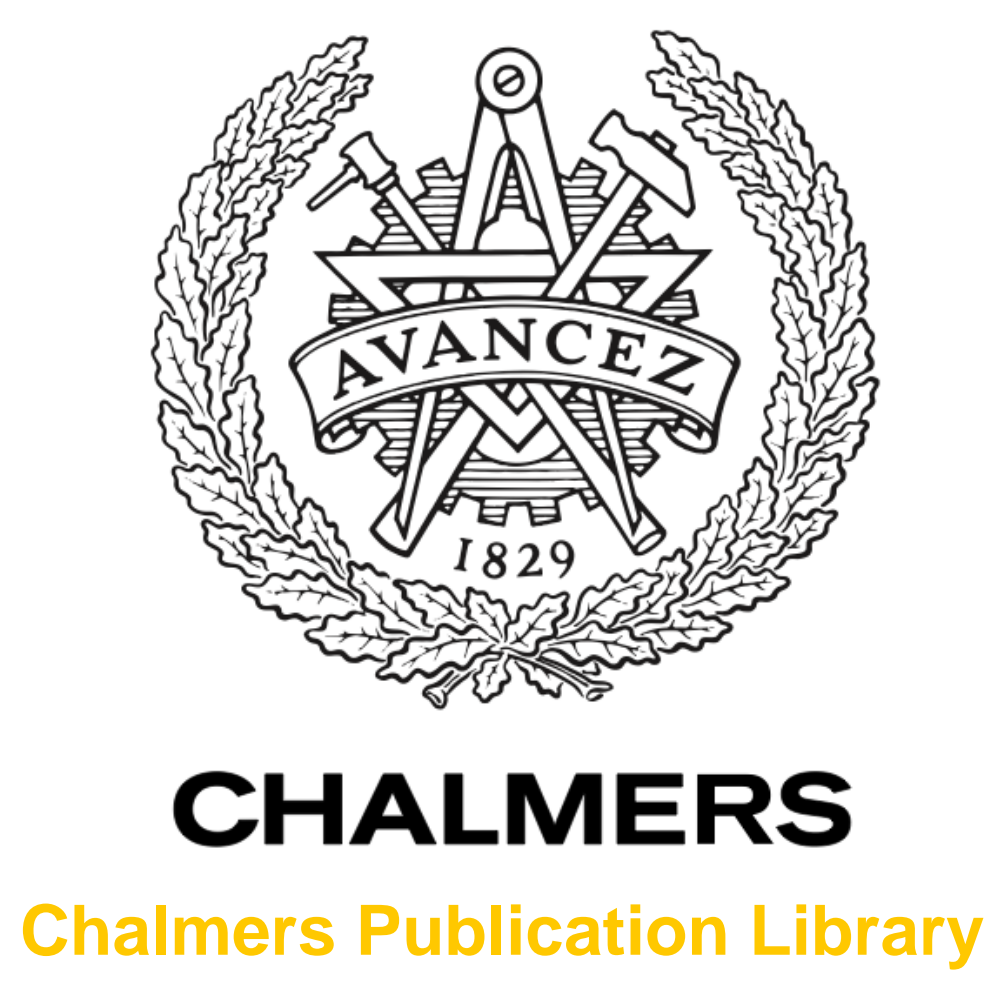

\author{
Error probability bounds for decode-and-forward relaying with two correlated \\ sources
}

This document has been downloaded from Chalmers Publication Library (CPL). It is the author's version of a work that was accepted for publication in:

IEEE Global Telecommunications Conference (GLOBECOM 2011), Houston, TX, USA (ISSN: 1930-529X)

Citation for the published paper:

Schwandter, S. ; Farès, H. ; Graell i Amat, A. (2011) "Error probability bounds for decodeand-forward relaying with two correlated sources". IEEE Global Telecommunications

Conference (GLOBECOM 2011), Houston, TX, USA pp. 1-5.

http://dx.doi.org/10.1109/GLOCOM.2011.6133984

Downloaded from: http://publications.lib.chalmers.se/publication/156928

Notice: Changes introduced as a result of publishing processes such as copy-editing and formatting may not be reflected in this document. For a definitive version of this work, please refer to the published source. Please note that access to the published version might require a subscription.

Chalmers Publication Library (CPL) offers the possibility of retrieving research publications produced at Chalmers University of Technology. It covers all types of publications: articles, dissertations, licentiate theses, masters theses, conference papers, reports etc. Since 2006 it is the official tool for Chalmers official publication statistics. To ensure that Chalmers research results are disseminated as widely as possible, an Open Access Policy has been adopted.

The CPL service is administrated and maintained by Chalmers Library. 


\title{
Error Probability Bounds for Decode-and-Forward Relaying with Two Correlated Sources
}

\author{
Stefan Schwandter ${ }^{\dagger}$, Haïfa Farès ${ }^{\ddagger}$, Alexandre Graell i Amat ${ }^{\S}$, and Gerald Matz ${ }^{\dagger}$ \\ ${ }^{\dagger}$ Institute for Telecommunications, Vienna University of Technology, Gusshausstrasse 25/389, A-1040 Vienna, Austria \\ $\ddagger$ Department of Electronics, Institut TELECOM-TELECOM Bretagne, 29238 Brest, France \\ $\S$ Department of Signals and Systems, Chalmers University of Technology, SE-412 96 Gothenburg, Sweden \\ Email: \{sschwand,gmatz\}@nt.tuwien.ac.at, haifa.fares@telecom-bretagne.eu, alexandre.graell@chalmers.se
}

\begin{abstract}
We derive bounds on the error probability of optimal and sub-optimal detectors in an uncoded decode-andforward relay system with two correlated information sources. This setup is relevant to wireless sensor networks where nearby sensors collect spatially correlated data. We show that taking into account the source correlation at the relay and at the destination leads to significant performance gains. Simulation results corroborate the tightness of our analytical bounds.
\end{abstract}

\section{INTRODUCTION}

We consider a wireless communication system with two sources that transmit their data to one common destination with the help of a relay (see Fig. 1). The data of the two sources is assumed to be correlated, an assumption that applies, for example, to wireless sensor networks (WSNs) [1], where nearby sensors measure statistically dependent data and transmit this data to a common processing node. We further restrict ourselves to uncoded transmission, which is practically relevant since power is a scarce resource in inexpensive sensor nodes and hence a coding/decoding stage may be infeasible.

The relay works in a half-duplex mode and uses the decodeand-forward (DF) strategy introduced and analyzed in [2]. The relay makes a hard decision on the transmitted data and forwards it to the destination. Instead of relaying the information of the individual sources in an alternating way, network coding [3] is used at the relay to combine the data of the two sources, thereby increasing the transmission capacity. The relay network in Fig. 1 was first considered in [4], where coding strategies were proposed. For the same relay network, performance bounds for convolutionally coded DF were derived in [5]. These works assumed that the data transmitted by the two sources is statistically independent. Joint detection of correlated sources (for a non-cooperative system) has been recently addressed in [6].

In this paper, we derive analytical bounds on the error probability for the relay system shown in Fig. 1. Our work extends the results in [5] by introducing correlation between the sources. We consider two types of detectors at the destination: i) the optimal maximum a posteriori (MAP) decoding

The work of S. Schwandter and G. Matz was funded by FWF Grant S10606 and WWTF Grant ICT08-44. A. Graell i Amat was supported by the Swedish Agency for Innovation Systems (VINNOVA) under the P36604-1 MAGIC project.

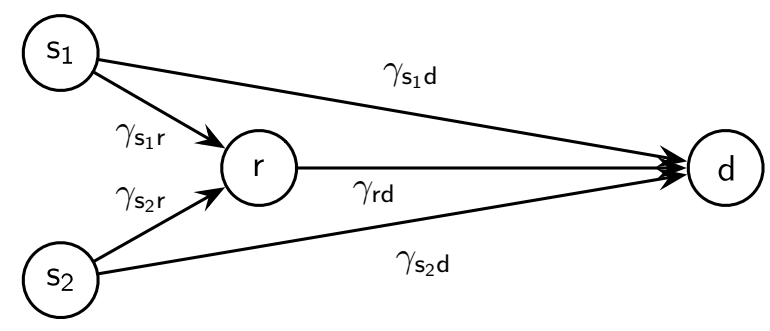

Fig. 1. Relay network consisting of two correlated sources with a common destination and one relay.

rule which explicitly takes into account that detection errors may occur at the relay; ii) a simplified MAP decoder that works on the assumption that the two-hop source-to-relay-todestination channel can be approximated by a virtual singlehop memoryless channel between the sources and the destination (this assumption has been previously used e.g. in [7]); the corresponding receiver will be termed the virtual-channel MAP (VC-MAP) decoder.

We demonstrate the performance gain that can be obtained by exploiting the correlation between the sources and we compare our results to a correlation-aware system without relay. It turns out that the gain achievable with a relay decreases with increasing correlation, i.e., for scenarios with very high correlation the gain is limited. Furthermore, we show that by tuning a free parameter in the sub-optimal detector the performance of the optimal detector can be closely approached. Simulation results confirm the tightness of the bounds obtained.

\section{SySTEM MODEL}

We consider the wireless relay channel with two sources, one relay and one destination depicted in Fig. 1. In practice, terminals cannot transmit and receive at the same time and over the same frequency band and hence we assume that all transmissions are over orthogonal channels. We restrict ourselves to uncoded binary transmission and BPSK modulation. Each source $\mathrm{s}_{i}, i=1,2$, wants to transmit an information bit $x_{\mathrm{s}_{i}} \in\{0,1\}$ to the destination (we assume $\left.p\left(x_{\mathrm{s}_{i}}=0\right)=p\left(x_{\mathrm{s}_{i}}=1\right)=0.5\right)$. The statistical dependence of the two source bits can be represented by the relation 
[6] $x_{\mathrm{s}_{2}}=x_{\mathrm{s}_{1}} \oplus a$, where $\oplus$ is the modulo- 2 addition; here, $a \in\{0,1\}$ is a random variable that is independent of $x_{\mathrm{s}_{1}}$ and $x_{\mathrm{s}_{2}}$ and determines the amount of correlation. We denote by $p_{a} \in[0.5,1]$ the probability that $a$ equals 0 $\left(p_{a}=0.5\right.$ corresponds to the case where $x_{\mathrm{s}_{1}}$ and $x_{\mathrm{s}_{2}}$ are uncorrelated). Note that $p\left(x_{\mathrm{s}_{1}}, x_{\mathrm{s}_{2}}\right)=p_{a} / 2$ for $x_{\mathrm{s}_{1}}=x_{\mathrm{s}_{2}}$ and $p\left(x_{\mathrm{s}_{1}}, x_{\mathrm{s}_{2}}\right)=\left(1-p_{a}\right) / 2$ for $x_{\mathrm{s}_{1}} \neq x_{\mathrm{s}_{2}}$.

The sources modulate their information bits $x_{\mathrm{s}_{i}}$ to BPSK symbols $\tilde{x}_{\mathrm{s}_{i}} \in\{ \pm 1\}$ using the mapping $0 \rightarrow+1,1 \rightarrow-1$ and broadcast these BPSK symbols to the relay and the destination, which receive the signals

$$
\begin{aligned}
y_{\mathrm{s}_{i} \mathrm{r}} & =h_{\mathrm{s}_{i} \mathrm{r}} \tilde{x}_{\mathrm{s}_{i}}+w_{\mathrm{s}_{i} \mathrm{r}} \\
y_{\mathrm{s}_{i} \mathrm{~d}} & =h_{\mathrm{s}_{i} \mathrm{~d}} \tilde{x}_{\mathrm{s}_{i}}+w_{\mathrm{s}_{i} \mathrm{~d}}, \quad i=1,2 .
\end{aligned}
$$

Here $w_{\mathrm{s}_{i} \mathrm{r}}$ and $w_{\mathrm{s}_{i} \mathrm{~d}}$ are additive white Gaussian noise (AWGN) variables with zero-mean and respective variance $\sigma_{\mathrm{s}_{i} \mathrm{r}}^{2}$ and $\sigma_{\mathrm{s}_{i} \mathrm{~d}}$, and $h_{\mathrm{s}_{i} \mathrm{r}}$ and $h_{\mathrm{s}_{i} \mathrm{~d}}$ denote the source-to-relay and sourceto-destination channel coefficients, respectively. Two different channel models are considered: binary-input AWGN $\left(h_{\mathrm{s}_{i} \mathrm{r}}=\right.$ $\left.h_{\mathrm{s}_{i} \mathrm{~d}}=1\right)$ and Rayleigh fading. In the fading case, each coefficient is a Rayleigh distributed, unit-variance random variable. The signal-to-noise ratio (SNR) for the source-to-relay and source-to-destination links are given by $\gamma_{\mathrm{s}_{i} \mathrm{r}}=1 /\left(2 \sigma_{\mathrm{s}_{i} \mathrm{r}}^{2}\right)$ and $\gamma_{\mathrm{s}_{i} \mathrm{~d}}=1 /\left(2 \sigma_{\mathrm{s}_{i} \mathrm{~d}}^{2}\right)$, respectively.

In order to exploit the correlation between the source symbols, the relay uses both $y_{\mathrm{s}_{1} \mathrm{r}}$ and $y_{\mathrm{s}_{2} \mathrm{r}}$ to jointly detect the source signals. The relay then forwards the modulo- 2 sum $x_{\mathrm{r}}=\hat{x}_{\mathrm{s}_{1} \mathrm{r}} \oplus \hat{x}_{\mathrm{s}_{2} \mathrm{r}}$ of the two detected signals $\hat{x}_{\mathrm{s}_{1} \mathrm{r}}$ and $\hat{x}_{\mathrm{S}_{2} \mathrm{r}}$ to the destination, which receives

$$
y_{\mathrm{rd}}=h_{\mathrm{rd}} \tilde{x}_{\mathrm{r}}+w_{\mathrm{rd}} .
$$

Here, $w_{\mathrm{rd}}$ is AWGN with variance $\sigma_{\mathrm{rd}}^{2}$ and $h_{\mathrm{rd}}$ is the relayto-destination channel coefficient which in case of a pure AWGN channel equals 1 and in case of fading has Rayleigh distribution with variance 1 . Correspondingly, the relay-todestination SNR equals $\gamma_{\mathrm{rd}}=1 /\left(2 \sigma_{\mathrm{rd}}^{2}\right)$. Since detection errors may occur at the relay, $x_{\mathrm{r}}$ may be different from $x_{\mathrm{s}_{1}} \oplus x_{\mathrm{s}_{2}}$. Based on the noisy observations $y_{\mathrm{s}_{1} \mathrm{~d}}, y_{\mathrm{s}_{2} \mathrm{~d}}$, and $y_{\mathrm{rd}}$, the destination attempts to detect $x_{\mathrm{s}_{1}}$ and $x_{\mathrm{s}_{2}}$. The detected symbols are denoted by $\hat{x}_{\mathrm{s}_{1}}$ and $\hat{x}_{\mathrm{s}_{2}}$.

\section{BOUNDS ON THE ERROR PROBABILITY}

The error event at the destination is defined by

$$
e=\left\{\hat{x}_{\mathrm{s}_{1}} \neq x_{\mathrm{s}_{1}} \vee \hat{x}_{\mathrm{s}_{2}} \neq x_{\mathrm{s}_{2}}\right\}
$$

For our analysis, we assume $x_{\mathrm{s}_{1}}=0$. This implies no loss of generality since $x_{\mathrm{S}_{1}}$ has a symmetric distribution.

Defining the error events at the relay,

$$
e_{\mathrm{r}_{1}}=\left\{\hat{x}_{\mathrm{s}_{1} \mathrm{r}} \neq x_{\mathrm{s}_{1}}\right\}, \quad e_{\mathrm{r}_{2}}=\left\{\hat{x}_{\mathrm{s}_{2} \mathrm{r}} \neq x_{\mathrm{s}_{2}}\right\},
$$

and

$$
e_{\mathrm{r}}=\left(e_{\mathrm{r}_{1}} \cup e_{\mathrm{r}_{2}}\right) \backslash\left(e_{\mathrm{r}_{1}} \cap e_{\mathrm{r}_{2}}\right) \text {, }
$$

the error probability at the destination can be written as

$$
\begin{aligned}
p(e)= & p\left(e \mid \bar{e}_{\mathrm{r}}, x_{\mathrm{s}_{1}}=x_{\mathrm{s}_{2}}\right) p\left(\bar{e}_{\mathrm{r}}, x_{\mathrm{s}_{1}}=x_{\mathrm{s}_{2}}\right) \\
& +p\left(e \mid \bar{e}_{\mathrm{r}}, x_{\mathrm{s}_{1}} \neq x_{\mathrm{s}_{2}}\right) p\left(\bar{e}_{\mathrm{r}}, x_{\mathrm{s}_{1}} \neq x_{\mathrm{s}_{2}}\right) \\
& +p\left(e \mid e_{\mathrm{r}}, x_{\mathrm{s}_{1}}=x_{\mathrm{s}_{2}}\right) p\left(e_{\mathrm{r}}, x_{\mathrm{s}_{1}}=x_{\mathrm{s}_{2}}\right) \\
& +p\left(e \mid e_{\mathrm{r}}, x_{\mathrm{s}_{1}} \neq x_{\mathrm{s}_{2}}\right) p\left(e_{\mathrm{r}}, x_{\mathrm{s}_{1}} \neq x_{\mathrm{s}_{2}}\right),
\end{aligned}
$$

where we distinguish between the four cases where the relay makes an error and where it does not, and where the source symbols are identical or not. We write $\bar{e}_{\mathrm{r}}$ for the complementary event of $e_{\mathrm{r}}$. For example, in the event $\left\{\bar{e}_{\mathrm{r}}, x_{\mathrm{s}_{1}}=x_{\mathrm{s}_{2}}\right\}, x_{\mathrm{r}}$ is equal to 0 .

For the detection at the relay we use a MAP detector that jointly detects $x_{\mathrm{s}_{1}}$ and $x_{\mathrm{s}_{2}}$ by taking into account the correlation between the sources according to

$$
\begin{aligned}
{\left[\hat{x}_{\mathrm{s}_{1} \mathrm{r}}, \hat{x}_{\mathrm{s}_{2} \mathrm{r}}\right]=} & \underset{\left[x_{\mathrm{s}_{1}}^{\prime}, x_{\mathrm{s}_{2}}^{\prime}\right]}{\operatorname{argmax}} p\left(x_{\mathrm{s}_{1}}^{\prime}, x_{\mathrm{s}_{2}}^{\prime} \mid y_{\mathrm{s}_{1} \mathrm{r}}, y_{\mathrm{s}_{2} \mathrm{r}}\right) \\
= & \underset{\left[x_{\mathrm{s}_{1}}^{\prime}, x_{\mathrm{s}_{2}}^{\prime}\right]}{\operatorname{argmax}} p\left(y_{\mathrm{s}_{1} \mathrm{r}} \mid x_{\mathrm{s}_{1}}^{\prime}\right) p\left(y_{\mathrm{s}_{2} \mathrm{r}} \mid x_{\mathrm{s}_{2}}^{\prime}\right) p\left(x_{\mathrm{s}_{1}}^{\prime}, x_{\mathrm{s}_{2}}^{\prime}\right) .
\end{aligned}
$$

Alternatively, we can write the MAP detector using L-values (log-likelihood ratios) as

$$
\left[\hat{x}_{\mathrm{s}_{1} \mathrm{r}}, \hat{x}_{\mathrm{s}_{2} \mathrm{r}}\right]=\underset{\left[x_{\mathrm{s}_{1}}, x_{\mathrm{s}_{2}}^{\prime}\right]}{\operatorname{argmax}}\left\{\bar{x}_{\mathrm{s}_{1}}^{\prime} L_{\mathrm{s}_{1} \mathrm{r}}+\bar{x}_{\mathrm{s}_{2}}^{\prime} L_{\mathrm{s}_{2} \mathrm{r}}+\ln p\left(x_{\mathrm{s}_{1}}^{\prime}, x_{\mathrm{s}_{2}}^{\prime}\right)\right\}
$$

where $\bar{x}=x \oplus 1$ and the L-values are defined as

$$
L_{\mathrm{s}_{1} \mathrm{r}}=\ln \frac{p\left(y_{\mathrm{s}_{1} \mathrm{r}} \mid x_{\mathrm{s}_{1} \mathrm{r}}=0\right)}{p\left(y_{\mathrm{s}_{1} \mathrm{r}} \mid x_{\mathrm{s}_{1} \mathrm{r}}=1\right)}=4 \gamma_{\mathrm{s}_{1} \mathrm{r}} y_{\mathrm{s}_{1} \mathrm{r}}, \quad L_{\mathrm{s}_{2} \mathrm{r}}=4 \gamma_{\mathrm{s}_{2} \mathrm{r}} y_{\mathrm{s}_{2} \mathrm{r}} .
$$

The relay error probabilities in (2) can be developed as

$p\left(\bar{e}_{\mathrm{r}}, x_{\mathrm{s}_{1}}=x_{\mathrm{s}_{2}}\right)=p\left(\bar{e}_{\mathrm{r}_{1}}, \bar{e}_{\mathrm{r}_{2}}, x_{\mathrm{s}_{1}}=x_{\mathrm{s}_{2}}\right)+p\left(e_{\mathrm{r}_{1}}, e_{\mathrm{r}_{2}}, x_{\mathrm{s}_{1}}=x_{\mathrm{s}_{2}}\right)$ $p\left(\bar{e}_{\mathrm{r}}, x_{\mathrm{s}_{1}} \neq x_{\mathrm{s}_{2}}\right)=p\left(\bar{e}_{\mathrm{r}_{1}}, \bar{e}_{\mathrm{r}_{2}}, x_{\mathrm{s}_{1}} \neq x_{\mathrm{s}_{2}}\right)+p\left(e_{\mathrm{r}_{1}}, e_{\mathrm{r}_{2}}, x_{\mathrm{s}_{1}} \neq x_{\mathrm{s}_{2}}\right)$ $p\left(e_{\mathrm{r}}, x_{\mathrm{s}_{1}}=x_{\mathrm{s}_{2}}\right)=p\left(\bar{e}_{\mathrm{r}_{1}}, e_{\mathrm{r}_{2}}, x_{\mathrm{s}_{1}}=x_{\mathrm{s}_{2}}\right)+p\left(e_{\mathrm{r}_{1}}, \bar{e}_{\mathrm{r}_{2}}, x_{\mathrm{s}_{1}}=x_{\mathrm{s}_{2}}\right)$ $p\left(e_{\mathrm{r}}, x_{\mathrm{s}_{1}} \neq x_{\mathrm{s}_{2}}\right)=p\left(\bar{e}_{\mathrm{r}_{1}}, e_{\mathrm{r}_{2}}, x_{\mathrm{s}_{1}} \neq x_{\mathrm{s}_{2}}\right)+p\left(e_{\mathrm{r}_{1}}, \bar{e}_{\mathrm{r}_{2}}, x_{\mathrm{s}_{1}} \neq x_{\mathrm{s}_{2}}\right)$.

From (3), after some basic calculations, the error probabilities at the relay for the case $x_{\mathrm{s}_{1}}=x_{\mathrm{s}_{2}}$ and an AWGN channel can be bounded as

$$
\begin{aligned}
& p\left(\bar{e}_{\mathrm{r}_{1}}, e_{\mathrm{r}_{2}}, x_{\mathrm{s}_{1}}=x_{\mathrm{s}_{2}}\right) \leq \frac{p_{a}}{2} \operatorname{erfc}\left(\frac{4 \gamma_{\mathrm{s}_{2} \mathrm{r}}-L_{a}}{4 \sqrt{\gamma_{\mathrm{s}_{2} \mathrm{r}}}}\right) \\
& p\left(e_{\mathrm{r}_{1}}, \bar{e}_{\mathrm{r}_{2}}, x_{\mathrm{s}_{1}}=x_{\mathrm{s}_{2}}\right) \leq \frac{p_{a}}{2} \operatorname{erfc}\left(\frac{4 \gamma_{\mathrm{s}_{1} \mathrm{r}}-L_{a}}{4 \sqrt{\gamma_{\mathrm{s}_{1} \mathrm{r}}}}\right) \\
& p\left(e_{\mathrm{r}_{1}}, e_{\mathrm{r}_{2}}, x_{\mathrm{s}_{1}}=x_{\mathrm{s}_{2}}\right) \leq \frac{p_{a}}{2} \operatorname{erfc}\left(\sqrt{\gamma_{\mathrm{s}_{1} \mathrm{r}}+\gamma_{\mathrm{s}_{2} \mathrm{r}}}\right) \\
& p\left(\bar{e}_{\mathrm{r}_{1}}, \bar{e}_{\mathrm{r}_{2}}, x_{\mathrm{s}_{1}}=x_{\mathrm{s}_{2}}\right) \leq p_{a},
\end{aligned}
$$

where

$$
L_{a}=\ln \frac{p\left(x_{\mathrm{s}_{2}} \neq x_{\mathrm{s}_{1}}\right)}{p\left(x_{\mathrm{s}_{2}}=x_{\mathrm{s}_{1}}\right)}=\ln \frac{1-p_{a}}{p_{a}} .
$$

The bounds for the case $x_{\mathrm{s}_{1}} \neq x_{\mathrm{S}_{2}}$ are obtained by replacing $p_{a}$ by $1-p_{a}$ and $L_{a}$ by $-L_{a}$ in (4)-(7).

Similar bounding expressions can be found for a Rayleigh fading channel. In this case, the SNRs in the expressions are random variables, and the bounds for the AWGN channel hold for a certain realization of these random variables. To 
obtain the average error probability for the fading channel, it is necessary to take the expection with respect to the joint distribution of the SNRs [8]. In the following, due to lack of space, we restrict the derivations to the AWGN channel.

\section{A. VC-MAP Decoder}

The VC-MAP decoder detects the symbols transmitted by the sources assuming that the observation $y_{\mathrm{rd}}$ is the output of a virtual memoryless channel with input $x_{\mathrm{s}_{12}}=x_{\mathrm{s}_{1}} \oplus x_{\mathrm{s}_{2}}$ and SNR $\gamma_{\mathrm{rd}}^{\prime} \leq \gamma_{\mathrm{rd}}$. Therefore, the VC-MAP implicitly models the relay decoding errors via a degraded SNR. Note that the degraded SNR $\gamma_{\text {rd }}^{\prime}$ can be tuned to optimize the VC-MAP performance. Based on this model, the VC-MAP detector reads

$$
\begin{aligned}
& {\left[\hat{x}_{s_{1}}, \hat{x}_{s_{2}}\right]=\underset{\left[x_{\mathrm{s}_{1}}, x_{\mathrm{s}_{2}}^{\prime}\right]}{\operatorname{argmax}} p\left(x_{\mathrm{s}_{1}}^{\prime}, x_{\mathrm{s}_{2}}^{\prime}, x_{\mathrm{s}_{12}}^{\prime} \mid y_{\mathrm{s}_{1} \mathrm{~d}}, y_{\mathrm{s}_{2} \mathrm{~d}}, y_{\mathrm{rd}}\right)} \\
& =\underset{\left[x_{\mathrm{s}_{1}}, x_{\mathrm{s}_{2}}^{\prime}\right]}{\operatorname{argmax}} p\left(y_{\mathrm{s}_{1} \mathrm{~d}}, y_{\mathrm{s}_{2} \mathrm{~d}}, y_{\mathrm{rd}} \mid x_{\mathrm{s}_{1}}^{\prime}, x_{\mathrm{s}_{2}}^{\prime}, x_{\mathrm{s}_{12}}^{\prime}\right) p\left(x_{\mathrm{s}_{1}}^{\prime}, x_{\mathrm{s}_{2}}^{\prime}\right) \\
& =\underset{\left[x_{\mathrm{s}_{1}}, x_{\mathrm{s}_{2}}^{\prime}\right]}{\operatorname{argmax}}\left\{\bar{x}_{\mathrm{s}_{1}}^{\prime} L_{\mathrm{s}_{1}}+\bar{x}_{\mathrm{s}_{2}}^{\prime} L_{\mathrm{s}_{2}}+\bar{x}_{\mathrm{s}_{12}}^{\prime} L_{\mathrm{r}}+\ln p\left(x_{\mathrm{s}_{1}}^{\prime}, x_{\mathrm{s}_{2}}^{\prime}\right)\right\},
\end{aligned}
$$

where $x_{\mathrm{s}_{12}}^{\prime}=x_{\mathrm{s}_{1}}^{\prime} \oplus x_{\mathrm{s}_{2}}^{\prime}, L_{\mathrm{s}_{i}}=4 \gamma_{\mathrm{s}_{i} \mathrm{~d}} y_{\mathrm{s}_{i} \mathrm{~d}}$ and $L_{\mathrm{r}}=4 \gamma_{\mathrm{rd}}^{\prime} y_{\mathrm{rd}}$. In the second equality we used the fact that $p\left(x_{\mathrm{s}_{1}}, x_{\mathrm{s}_{2}}, x_{\mathrm{s}_{12}}\right)=$ $p\left(x_{\mathrm{s}_{1}}, x_{\mathrm{s}_{2}}\right)$, since $x_{\mathrm{s}_{12}}=x_{\mathrm{s}_{1}} \oplus x_{\mathrm{s}_{2}}$. Given $\tilde{x}_{\mathrm{s}_{i}}= \pm 1, L_{\mathrm{s}_{i}}$ has mean $\pm 4 \gamma_{\mathrm{s}_{i} \mathrm{~d}}$ and variance $8 \gamma_{\mathrm{s}_{i} \mathrm{~d}}$; given $\tilde{x}_{r}= \pm 1, L_{\mathrm{r}}$ has mean $\pm 4 \gamma_{\mathrm{rd}}^{\prime}$ and variance $8 \gamma_{\mathrm{rd}}^{\prime 2} / \gamma_{\mathrm{rd}}$. We next bound the four conditional error probabilities in (2).

Case $1\left(\bar{e}_{\mathrm{r}}, x_{\mathrm{s}_{1}}=x_{\mathrm{s}_{2}}\right)$ : This case is equivalent to the transmission of $x_{\mathrm{s}_{1}}, x_{\mathrm{s}_{2}}$ and $x_{\mathrm{r}}=0$ over three independent parallel channels with SNR $\gamma_{\mathrm{s}_{1} \mathrm{~d}}, \gamma_{\mathrm{s}_{2} \mathrm{~d}}$, and $\gamma_{\mathrm{rd}}^{\prime}$, respectively. Thus, the probability of error can be upper-bounded as (we define $\left.\mathbf{x}_{s}^{\prime}=\left[x_{\mathrm{s}_{1}}^{\prime}, x_{\mathrm{s}_{2}}^{\prime}\right]\right)$

$$
\begin{aligned}
& p\left(e \mid \bar{e}_{\mathrm{r}}, x_{\mathrm{s}_{1}}=x_{\mathrm{s}_{2}}\right)=p\left(e \mid x_{\mathrm{s}_{1}}=0, x_{\mathrm{s}_{2}}=0, x_{\mathrm{r}}=0\right) \\
& \leq \sum_{\mathbf{x}_{s}^{\prime} \neq[0,0]} p\left(\bar{x}_{\mathrm{s}_{1}}^{\prime} L_{\mathrm{s}_{1}}+\bar{x}_{\mathrm{s}_{2}}^{\prime} L_{\mathrm{s}_{2}}+\bar{x}_{\mathrm{s}_{12}}^{\prime} L_{\mathrm{r}}<\ln \frac{p\left(\mathbf{x}_{s}^{\prime}\right)}{p(0,0)}\right) \\
& =\frac{1}{2} \operatorname{erfc}\left(\frac{4\left(\gamma_{\mathrm{s}_{2} \mathrm{~d}}+\gamma_{\mathrm{rd}}^{\prime}\right)-L_{a}}{4 \sqrt{\gamma_{\mathrm{s}_{2} \mathrm{~d}}+\gamma_{\mathrm{rd}}^{\prime 2} / \gamma_{\mathrm{rd}}}}\right) \\
& \quad+\frac{1}{2} \operatorname{erfc}\left(\frac{4\left(\gamma_{\mathrm{s}_{1} \mathrm{~d}}+\gamma_{\mathrm{rd}}^{\prime}\right)-L_{a}}{4 \sqrt{\gamma_{\mathrm{s}_{1} \mathrm{~d}}+\gamma_{\mathrm{rd}}^{\prime 2} / \gamma_{\mathrm{rd}}}}\right) \\
& \quad+\frac{1}{2} \operatorname{erfc}\left(\sqrt{\gamma_{\mathrm{s}_{1} \mathrm{~d}}+\gamma_{\mathrm{s}_{2} \mathrm{~d}}}\right) .
\end{aligned}
$$

The last expression is obtained by observing that $L_{\mathrm{S}_{1}}, L_{\mathrm{s}_{2}}$ and $L_{\mathrm{r}}$ have positive mean. Therefore, the random variable $\bar{x}_{\mathrm{s}_{1}}^{\prime} L_{\mathrm{s}_{1}}+\bar{x}_{\mathrm{s}_{2}}^{\prime} L_{\mathrm{s}_{2}}+\bar{x}_{\mathrm{s}_{12}}^{\prime} L_{\mathrm{r}}$ is Gaussian with mean $4\left(\bar{x}_{\mathrm{s}_{1}}^{\prime} \gamma_{\mathrm{s}_{1} \mathrm{~d}}+\bar{x}_{\mathrm{s}_{2},}^{\prime} \gamma_{\mathrm{s}_{2} \mathrm{~d}}+\bar{x}_{\mathrm{s}_{12}}^{\prime} \gamma_{\mathrm{rd}}^{\prime}\right)$ and variance $8\left(\bar{x}_{\mathrm{s}_{1}}^{\prime} \gamma_{\mathrm{s}_{1} \mathrm{~d}}+\right.$ $\left.\bar{x}_{\mathrm{s}_{2}}^{\prime} \gamma_{\mathrm{s}_{2} \mathrm{~d}}+\bar{x}_{\mathrm{s}_{12}}^{\prime} \gamma_{\mathrm{rd}}^{\prime} / \gamma_{\mathrm{rd}}\right)$

Case $2\left(\bar{e}_{\mathrm{r}}, x_{\mathrm{s}_{1}} \neq x_{\mathrm{s}_{2}}\right)$ : A bound for the probability $p\left(e \mid \bar{e}_{\mathrm{r}}, x_{\mathrm{s}_{1}} \neq x_{\mathrm{s}_{2}}\right)$ is obtained by replacing $L_{a}$ by $-L_{a}$ in (9).

Case $3\left(e_{\mathrm{r}}, x_{\mathrm{s}_{1}}=x_{\mathrm{s}_{2}}\right)$ : In this case, the relay decodes one of the sources with error, i.e., $x_{\mathrm{r}}=1$. The probability $p\left(e \mid e_{\mathrm{r}}, x_{\mathrm{s}_{1}}=x_{\mathrm{s}_{2}}\right)$ can be upper-bounded as

$$
\begin{aligned}
p\left(e \mid e_{\mathrm{r}}, x_{\mathrm{s}_{1}}=x_{\mathrm{s}_{2}}\right)= & p\left(e \mid x_{\mathrm{s}_{1}}=0, x_{\mathrm{s}_{2}}=0, x_{\mathrm{r}}=1\right) \\
\leq & \frac{1}{2} \operatorname{erfc}\left(\frac{4\left(\gamma_{\mathrm{s}_{2} \mathrm{~d}}-\gamma_{\mathrm{rd}}^{\prime}\right)-L_{a}}{4 \sqrt{\gamma_{\mathrm{s}_{2} \mathrm{~d}}+\gamma_{\mathrm{rd}}^{\prime 2} / \gamma_{\mathrm{rd}}}}\right) \\
& +\frac{1}{2} \operatorname{erfc}\left(\frac{4\left(\gamma_{\mathrm{s}_{1} \mathrm{~d}}-\gamma_{\mathrm{rd}}^{\prime}\right)-L_{a}}{4 \sqrt{\gamma_{\mathrm{s}_{1} \mathrm{~d}}+\gamma_{\mathrm{rd}}^{\prime 2} / \gamma_{\mathrm{rd}}}}\right) \\
& +\frac{1}{2} \operatorname{erfc}\left(\sqrt{\gamma_{\mathrm{s}_{1} \mathrm{~d}}+\gamma_{\mathrm{s}_{2} \mathrm{~d}}}\right)
\end{aligned}
$$

The last expression is obtained by observing that $L_{\mathrm{s}_{1}}$ and $L_{\mathrm{s}_{2}}$ have positive mean while $L_{\mathrm{r}}$ has negative mean due to the error event at the relay. Therefore, $\bar{x}_{\mathrm{s}_{1}}^{\prime} L_{\mathrm{s}_{1}}+\bar{x}_{\mathrm{s}_{2}}^{\prime} L_{\mathrm{s}_{2}}+\bar{x}_{\mathrm{s}_{12}}^{\prime} L_{\mathrm{r}}$ is Gaussian with mean $4\left(\bar{x}_{\mathrm{s}_{1}}^{\prime} \gamma_{\mathrm{s}_{1} \mathrm{~d}}+\bar{x}_{\mathrm{s}_{2}}^{\prime} \gamma_{\mathrm{s}_{2} \mathrm{~d}}-\bar{x}_{\mathrm{s}_{12}}^{\prime} \gamma_{\mathrm{rd}}^{\prime}\right)$ and variance $8\left(\bar{x}_{\mathrm{s}_{1}}^{\prime} \gamma_{\mathrm{s}_{1} \mathrm{~d}}+\bar{x}_{\mathrm{s}_{2}}^{\prime} \gamma_{\mathrm{s}_{2} \mathrm{~d}}+\bar{x}_{\mathrm{s}_{12}}^{\prime} \gamma_{\mathrm{rd}}^{\prime} / \gamma_{\mathrm{rd}}\right)$.

Case $4\left(e_{\mathrm{r}}, x_{\mathrm{S}_{1}} \neq x_{\mathrm{S}_{2}}\right)$ : A bound for the probability $p\left(e \mid e_{\mathrm{r}}, x_{\mathrm{s}_{1}} \neq x_{\mathrm{s}_{2}}\right)$ is obtained by replacing $L_{a}$ (10) with $-L_{a}$.

Optimization of $\gamma_{\mathrm{rd}}^{\prime}$ : The virtual source-to-destination channel is clearly only an approximation since it subsumes the channel quality on the source-to-relay and relay-to-destination links and the detection errors at the relay into an equivalent Gaussian model characterized by the degraded SNR $\gamma_{\text {rd }}^{\prime}$. The SNR $\gamma_{\text {rd }}^{\prime}$ can be tuned such that the virtual channel approximation best matches the actual two-hop channel involving the source-to-relay and relay-to-destination links. Such an optimization of the SNR of the equivalent channel has been proposed in [5]. We will show via simulations in Section IV that this optimization is feasible also for the case of correlated sources and that it allows the VC-MAP detector to closely approach the performance of the MAP detector. The optimal $\gamma_{\text {rd }}^{\prime}$ can be calculated off-line before deployment.

\section{B. MAP Decoder}

In this section, we consider the MAP decoding rule, which is optimal for the scenario in Fig. 1 and is given by

$$
\begin{aligned}
{\left[\hat{x}_{s_{1}}, \hat{x}_{s_{2}}\right] } & =\underset{\left[x_{\mathrm{s}_{1}}^{\prime}, x_{\mathrm{s}_{2}}^{\prime}\right]}{\operatorname{argmax}} p\left(x_{\mathrm{s}_{1}}^{\prime}, x_{\mathrm{s}_{2}}^{\prime} \mid y_{\mathrm{s}_{1} \mathrm{~d}}, y_{\mathrm{s}_{2} \mathrm{~d}}, y_{\mathrm{rd}}\right) \\
& =\underset{\left[x_{\mathrm{s}_{1}}^{\prime}, x_{\mathrm{s}_{2}}^{\prime}\right]}{\operatorname{argmax}} \sum_{x_{\mathrm{r}} \in\{0,1\}} p\left(x_{\mathrm{s}_{1}}^{\prime}, x_{\mathrm{s}_{2}}^{\prime}, x_{\mathrm{r}} \mid y_{\mathrm{s}_{1} \mathrm{~d}}, y_{\mathrm{s}_{2} \mathrm{~d}}, y_{\mathrm{rd}}\right) .
\end{aligned}
$$

Since the evaluation of (11) requires a marginalization with respect to $x_{\mathrm{r}}$, we resort to a simplified approach that jointly estimates source and relay symbols. The corresponding (joint) MAP decoding rule reads

$$
\begin{aligned}
& {\left[\hat{x}_{\mathrm{s}_{1}}, \hat{x}_{\mathrm{s}_{2}}, \hat{x}_{\mathrm{r}}\right]=\underset{\left[x_{\mathrm{s}_{1}}^{\prime}, x_{\mathrm{s}_{2}}^{\prime}, x_{\mathrm{r}}^{\prime}\right]}{\operatorname{argmax}} p\left(x_{\mathrm{s}_{1}}^{\prime}, x_{\mathrm{s}_{2}}^{\prime}, x_{\mathrm{r}}^{\prime} \mid y_{\mathrm{s}_{1} \mathrm{~d}}, y_{\mathrm{s}_{2} \mathrm{~d}}, y_{\mathrm{rd}}\right)} \\
& =\underset{\left[x_{\mathrm{s}_{1}}^{\prime}, x_{\mathrm{s}_{2}}^{\prime}, x_{\mathrm{r}}^{\prime}\right]}{\operatorname{argmax}} p\left(y_{\mathrm{s}_{1} \mathrm{~d}}, y_{\mathrm{s}_{2} \mathrm{~d}}, y_{\mathrm{rd}} \mid x_{\mathrm{s}_{1}}^{\prime}, x_{\mathrm{s}_{2}}^{\prime}, x_{\mathrm{r}}^{\prime}\right) p\left(x_{\mathrm{s}_{1}}^{\prime}, x_{\mathrm{s}_{2}}^{\prime}, x_{\mathrm{r}}^{\prime}\right) \\
& =\underset{\left[x_{\mathrm{s}_{1}}^{\prime}, x_{\mathrm{s}_{2}}^{\prime}, x_{\mathrm{r}}^{\prime}\right]}{\operatorname{argmax}}\left\{\bar{x}_{\mathrm{s}_{1}}^{\prime} L_{\mathrm{s}_{1}}+\bar{x}_{\mathrm{s}_{2}}^{\prime} L_{\mathrm{s}_{2}}+\bar{x}_{\mathrm{r}}^{\prime} L_{\mathrm{r}}\right. \\
& \left.\quad+\ln p\left(x_{\mathrm{r}}^{\prime} \mid x_{\mathrm{s}_{1}}^{\prime}, x_{\mathrm{s}_{2}}^{\prime}\right)+\ln p\left(x_{\mathrm{s}_{1}}^{\prime}, x_{\mathrm{s}_{2}}^{\prime}\right)\right\} .
\end{aligned}
$$

Contrary to the VC-MAP rule, the fourth term in the last expression explicitly takes into account relay errors. 
From this decision rule, bounds on the error probability of the (joint) MAP detector can again be derived based on the expansion (2) of the total error probability. We follow a similar procedure as with the VC-MAP decoder and as in [9]. In our derivations below, we will use the following definitions:

$$
\begin{array}{cl}
L_{s}=\ln \frac{p\left(x_{\mathrm{r}}=0 \mid x_{\mathrm{s}_{1}}=x_{\mathrm{s}_{2}}\right)}{p\left(x_{\mathrm{r}}=1 \mid x_{\mathrm{s}_{1}}=x_{\mathrm{s}_{2}}\right)}, & L_{t}=\ln \frac{p\left(x_{\mathrm{r}}=0 \mid x_{\mathrm{s}_{1}} \neq x_{\mathrm{s}_{2}}\right)}{p\left(x_{\mathrm{r}}=1 \mid x_{\mathrm{s}_{1}} \neq x_{\mathrm{s}_{2}}\right)}, \\
L_{u}=\ln \frac{p\left(x_{\mathrm{r}}=0 \mid x_{\mathrm{s}_{1}}=x_{\mathrm{s}_{2}}\right)}{p\left(x_{\mathrm{r}}=0 \mid x_{\mathrm{s}_{1}} \neq x_{\mathrm{s}_{2}}\right)}, & L_{v}=\ln \frac{p\left(x_{\mathrm{r}}=1 \mid x_{\mathrm{s}_{1}}=x_{\mathrm{s}_{2}}\right)}{p\left(x_{\mathrm{r}}=1 \mid x_{\mathrm{s}_{1}} \neq x_{\mathrm{s}_{2}}\right)}, \\
L_{w}=\ln \frac{p\left(x_{\mathrm{r}}=0 \mid x_{\mathrm{s}_{1}}=x_{\mathrm{s}_{2}}\right)}{p\left(x_{\mathrm{r}}=1 \mid x_{\mathrm{s}_{1}} \neq x_{\mathrm{s}_{2}}\right)}, & L_{x}=\ln \frac{p\left(x_{\mathrm{r}}=0 \mid x_{\mathrm{s}_{1}} \neq x_{\mathrm{s}_{2}}\right)}{p\left(x_{\mathrm{r}}=1 \mid x_{\mathrm{s}_{1}}=x_{\mathrm{s}_{2}}\right)} .
\end{array}
$$

Case $1\left(\bar{e}_{\mathrm{r}}, x_{\mathrm{s}_{1}}=x_{\mathrm{s}_{2}}\right)$ : In this case, the probability of error can be upper-bounded as

$$
\begin{aligned}
p\left(e \mid \bar{e}_{\mathrm{r}},\right. & \left.x_{\mathrm{s}_{1}}=x_{\mathrm{s}_{2}}\right) \\
\leq & \frac{1}{2} \operatorname{erfc}\left(\frac{4 \gamma_{\mathrm{s}_{1} \mathrm{~d}}+L_{u}-L_{a}}{4 \sqrt{\gamma_{\mathrm{s}_{1} \mathrm{~d}}}}\right)+\operatorname{erfc}\left(\frac{4 \gamma_{\mathrm{s}_{2} \mathrm{~d}}+L_{u}-L_{a}}{4 \sqrt{\gamma_{\mathrm{s}_{2} \mathrm{~d}}}}\right) \\
& +\frac{1}{2} \operatorname{erfc}\left(\frac{4\left(\gamma_{\mathrm{s}_{1} \mathrm{~d}}+\gamma_{\mathrm{rd}}\right)+L_{w}-L_{a}}{4 \sqrt{\gamma_{\mathrm{s}_{1} \mathrm{~d}}+\gamma_{\mathrm{rd}}}}\right) \\
& +\frac{1}{2} \operatorname{erfc}\left(\frac{4\left(\gamma_{\mathrm{s}_{2} \mathrm{~d}}+\gamma_{\mathrm{rd}}\right)+L_{w}-L_{a}}{4 \sqrt{\gamma_{\mathrm{s}_{2} \mathrm{~d}}+\gamma_{\mathrm{rd}}}}\right) \\
& +\frac{1}{2} \operatorname{erfc}\left(\sqrt{\gamma_{\mathrm{s}_{1} \mathrm{~d}}+\gamma_{\mathrm{s}_{2} \mathrm{~d}}}\right) \\
& +\frac{1}{2} \operatorname{erfc}\left(\frac{4\left(\gamma_{\mathrm{s}_{1} \mathrm{~d}}+\gamma_{\mathrm{s}_{2} \mathrm{~d}}+\gamma_{\mathrm{rd}}\right)+L_{s}}{4 \sqrt{\gamma_{\mathrm{s}_{1} \mathrm{~d}}+\gamma_{\mathrm{s}_{2} \mathrm{~d}}+\gamma_{\mathrm{rd}}}}\right) .
\end{aligned}
$$

Case $2\left(\bar{e}_{\mathrm{r}}, x_{\mathrm{s}_{1}} \neq x_{\mathrm{s}_{2}}\right)$ : The bound here is obtained by replacing $L_{u}, L_{w}, L_{s}$, and $-L_{a}$ in (12) with $-L_{w},-L_{v}$, $-L_{t}$, and $L_{a}$, respectively.

Case $3\left(e_{\mathrm{r}}, x_{\mathrm{s}_{1}}=x_{\mathrm{s}_{2}}\right)$ : We have

$$
\begin{aligned}
p\left(e \mid e_{\mathrm{r}},\right. & \left.x_{\mathrm{s}_{1}}=x_{\mathrm{s}_{2}}\right) \\
\leq & \frac{1}{2} \operatorname{erfc}\left(\frac{4 \gamma_{\mathrm{s}_{1} \mathrm{~d}}+L_{v}-L_{a}}{4 \sqrt{\gamma_{\mathrm{s}_{1} \mathrm{~d}}}}\right)+\operatorname{erfc}\left(\frac{4 \gamma_{\mathrm{s}_{2} \mathrm{~d}}+L_{v}-L_{a}}{4 \sqrt{\gamma_{\mathrm{s}_{2} \mathrm{~d}}}}\right) \\
& +\frac{1}{2} \operatorname{erfc}\left(\frac{4\left(\gamma_{\mathrm{s}_{1} \mathrm{~d}}+\gamma_{\mathrm{rd}}\right)-L_{x}-L_{a}}{4 \sqrt{\gamma_{\mathrm{s}_{1} \mathrm{~d}}+\gamma_{\mathrm{rd}}}}\right) \\
& +\frac{1}{2} \operatorname{erfc}\left(\frac{4\left(\gamma_{\mathrm{s}_{2} \mathrm{~d}}+\gamma_{\mathrm{rd}}\right)-L_{x}-L_{a}}{4 \sqrt{\gamma_{\mathrm{s}_{2} \mathrm{~d}}+\gamma_{\mathrm{rd}}}}\right) \\
& +\frac{1}{2} \operatorname{erfc}\left(\sqrt{\gamma_{\mathrm{s}_{1} \mathrm{~d}}+\gamma_{\mathrm{s}_{2} \mathrm{~d}}}\right) \\
& +\frac{1}{2} \operatorname{erfc}\left(\frac{4\left(\gamma_{\mathrm{s}_{1} \mathrm{~d}}+\gamma_{\mathrm{s}_{2} \mathrm{~d}}+\gamma_{\mathrm{rd}}\right)-L_{s}}{4 \sqrt{\gamma_{\mathrm{s}_{1} \mathrm{~d}}+\gamma_{\mathrm{s}_{2} \mathrm{~d}}+\gamma_{\mathrm{rd}}}}\right) .
\end{aligned}
$$

Case $4\left(e_{\mathrm{r}}, x_{\mathrm{s}_{1}} \neq x_{\mathrm{s}_{2}}\right)$ : The error bound here is obtained by replacing $L_{v}, L_{x},-L_{s}$, and $-L_{a}$ in (13) with $-L_{u}, L_{v}$, $L_{t}$, and $L_{a}$, respectively.

\section{NumericAl Results}

In this section, we illustrate the tightness of the error probability bounds derived in Sections III-A and III-B by comparing them to simulation results. We also compare the performance of the VC-MAP decoder and the MAP decoder. For simplicity, we assume that the SNRs are symmetric, i.e.,

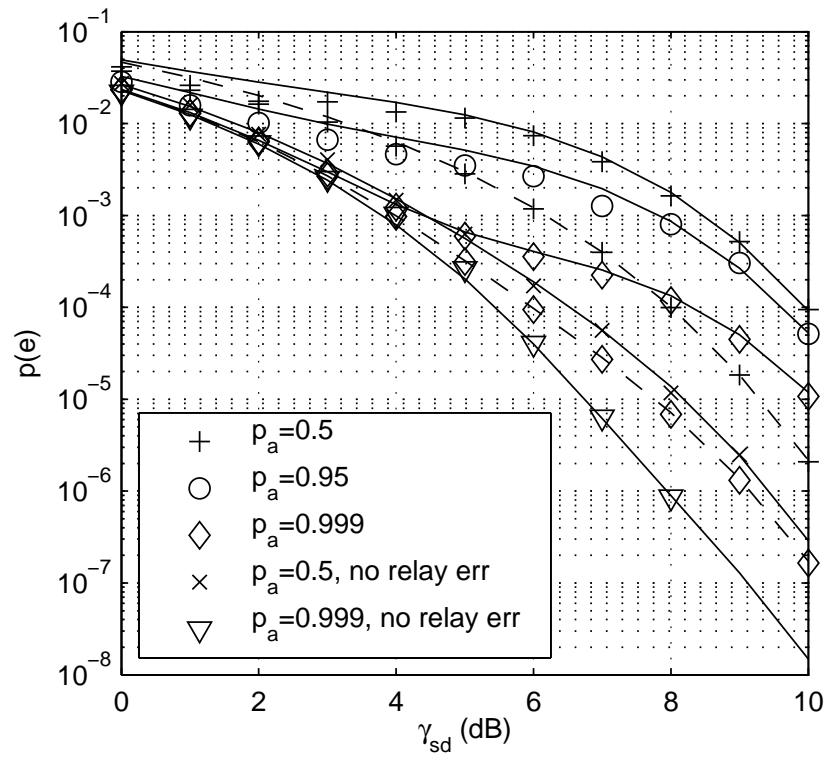

Fig. 2. Error probability bounds (lines) and simulations (markers) of a relay system with two correlated sources for a VC-MAP decoder with AWGN channels for the cases $\gamma_{\mathrm{rd}}^{\prime}=\gamma_{\mathrm{rd}}$ (solid lines) and $\gamma_{\mathrm{rd}}^{\prime}=\gamma_{\mathrm{opt}}$ (dashed lines); here, $\gamma_{\mathrm{sr}}=\gamma_{\mathrm{rd}}=5 \mathrm{~dB}$.

$\gamma_{\mathrm{s}_{1} \mathrm{~d}}=\gamma_{\mathrm{s}_{2} \mathrm{~d}}=\gamma_{\mathrm{sd}}$ and $\gamma_{\mathrm{s}_{1} \mathrm{r}}=\gamma_{\mathrm{s}_{2} \mathrm{r}}=\gamma_{\mathrm{sr}}$. We also consider fixed $\gamma_{\mathrm{sr}}$ and $\gamma_{\mathrm{rd}}$.

In Fig. 2, we plot the bounds on the error probability for the VC-MAP decoder for $\gamma_{\mathrm{rd}}^{\prime}=\gamma_{\mathrm{rd}}$ (solid lines) and for $\gamma_{\mathrm{rd}}^{\prime}=\gamma_{\mathrm{opt}}$ (dashed lines) together with the simulation results (markers), as a function of $\gamma_{\mathrm{sd}}$ (in $\mathrm{dB}$ ), for several degrees of correlation between the two sources, $\gamma_{\mathrm{sr}}=\gamma_{\mathrm{rd}}=5 \mathrm{~dB}$, and AWGN channels. A good match between the simulations and the bounds is observed. We note that $\gamma_{\mathrm{rd}}^{\prime}=\gamma_{\mathrm{rd}}$ yields a significant deviation from the case where the source-torelay channels are error-free. However, for optimized $\gamma_{\mathrm{rd}}^{\prime}$ $\left(\gamma_{\mathrm{rd}}^{\prime}=\gamma_{\mathrm{opt}}\right)$, the bounds are much closer to the case of errorfree source-to-relay channels.

Fig. 3 shows the bounds on the error probability (lines), together with the corresponding simulation results (markers) as a function of $\gamma_{\mathrm{sd}}^{b}$, where $\gamma_{\mathrm{sd}}^{b}=\gamma_{\mathrm{sd}} / R$, for the VC-MAP decoder over a Rayleigh channel for both a non-cooperative system and a single relay cooperative system. The system rate equals $R=1$ and $R=2 / 3$, respectively, for the case of no relay (dashed lines) and one relay (solid lines). In the figure, $\gamma_{\mathrm{rd}}^{\prime}=\gamma_{\mathrm{opt}}$ and $\gamma_{\mathrm{sr}}=\gamma_{\mathrm{rd}}=15 \mathrm{~dB}$. While performance is generally better for more strongly correlated sources, the relay is less useful in this situation.

Fig. 4 shows the bounds on the error probability (solid lines) of the (joint) MAP detector, as well as the corresponding simulation results (markers) as a function of $\gamma_{\mathrm{sd}}^{b}$ for Rayleigh fading. Sizable gains with increasing level of correlation (increasing $p_{a}$ ) are observed over the uncorrelated case $\left(p_{a}=0.5\right)$. The latter case corresponds to the scenario where there is no source correlation or where a demodulator oblivious of existing correlation is used. For example, the gain for $p_{a}=0.95$ is over $5 \mathrm{~dB}$ at an error probability of $10^{-4}$. 


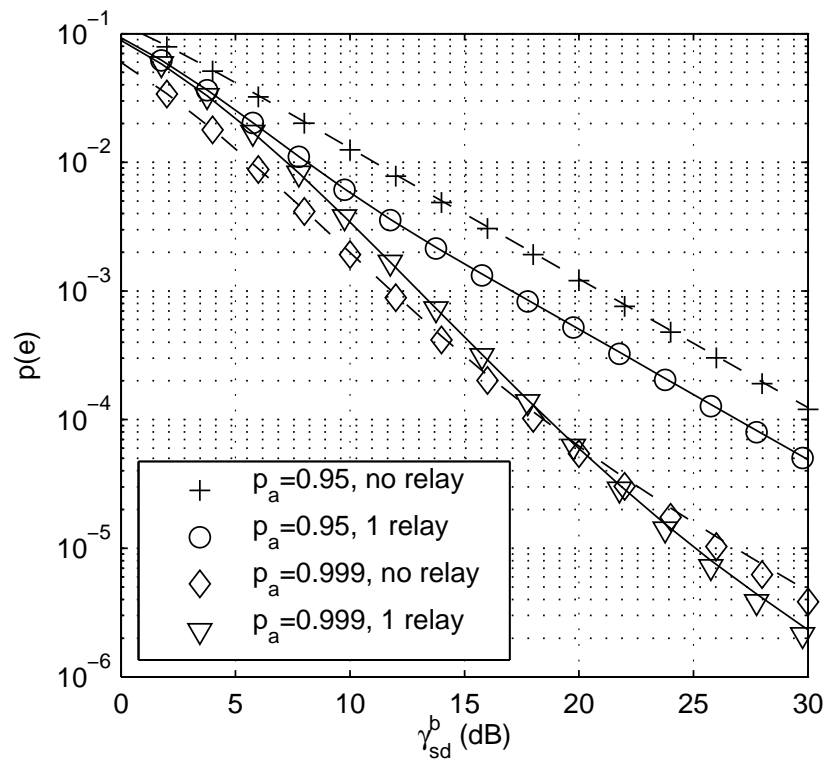

Fig. 3. Error probability bounds (lines) and simulations (markers) of a relay system with two correlated sources for a VC-MAP decoder in Rayleigh fading for two cases: non-cooperative system (dashed lines) and single relay cooperative system (solid lines); here, $\gamma_{\mathrm{sr}}=\gamma_{\mathrm{rd}}=15 \mathrm{~dB}$.

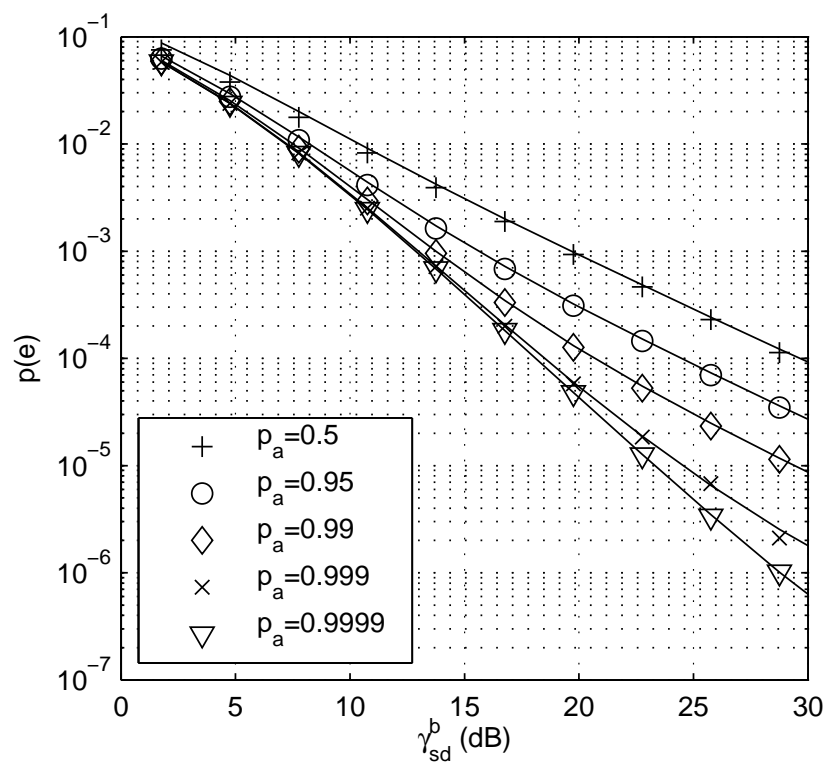

Fig. 4. Error probability bounds (lines) and simulations (markers) of a relay system with two correlated sources for a MAP decoder in Rayleigh fading for different correlation factors $p_{a}$; here, $\gamma_{\mathrm{sr}}=\gamma_{\mathrm{rd}}=15 \mathrm{~dB}$.

Fig. 5 shows the performance improvement that is achieved by the use of relaying and (joint) MAP detection over a scenario without cooperation (again for Rayleigh fading). With $p_{a}=0.95$, we observe a gain of about $6 \mathrm{~dB}$ at a $p(e)$ of $10^{-4}$. However, the gain vanishes for very high correlation $\left(p_{a}=0.999\right)$. Finally, comparing Fig. 3 with Fig. 5, we observe that the (lower-complexity) VC-MAP detector with optimized $\gamma_{\mathrm{rd}}^{\prime}$ approaches the performance of the (optimal) MAP detector very closely.

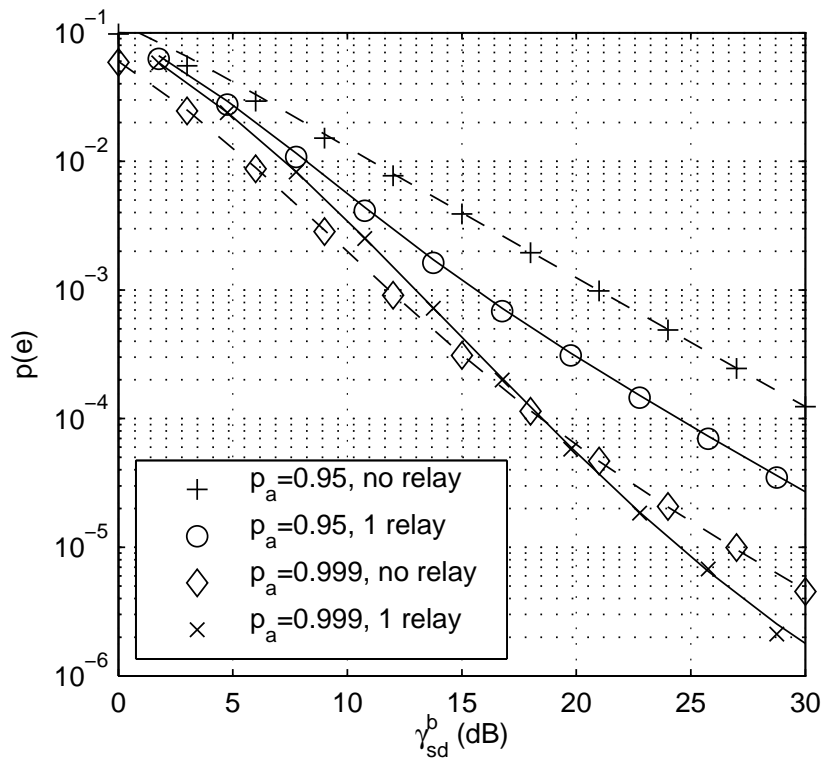

Fig. 5. Error probability bounds (lines) and simulations (markers) of a relay system with two correlated sources for a MAP decoder in Rayleigh fading for two cases: non-cooperative system (dashed lines) and single relay cooperative system (solid lines); here, $\gamma_{\mathrm{sr}}=\gamma_{\mathrm{rd}}=15 \mathrm{~dB}$.

\section{CONCLUSIONS}

We derived analytical bounds on the error probability of a decode-and-forward relaying system consisting of two correlated sources, one relay, and one destination. We showed that by taking into account the source correlation via joint channel detection at the relay and at the destination, noticeable performance gains can be achieved. We also showed that the use of a relay results in a performance improvement over a system without relay, specifically at medium correlation levels.

Future work includes the extension of our results to scenarios with more than two sources, more than one relay, higherorder modulation, and coded transmissions.

\section{REFERENCES}

[1] I. F. Akyildiz, W. Su, Y. Sankarasubramaniam, and E. Cayirci, "Wireless sensor networks: a survey," Computer Networks, vol. 38, no. 4, pp. 393422, 2002.

[2] T. Cover and A. E. Gamal, "Capacity theorems for the relay channel," IEEE Trans. Inf. Theory, vol. 25, pp. 572-584, Sep. 1979.

[3] R. Ahlswede, N. Cai, S. Li, and R. Yeung, "Network information flow," IEEE Trans. Inf. Theory, vol. 46, no. 4, pp. 1204-1216, Jul. 2000.

[4] C. Hausl and P. Dupraz, "Joint network-channel coding for the multipleaccess relay channel," in Proc. IEEE Commun. Society on Sensor and Ad Hoc Commun. and Networks (SECON), Sep. 2006, pp. 817-822.

[5] A. Graell i Amat and I. Land, "Bounds of the probability of error for decode-and-forward relaying with two sources," in Proc. IEEE Symp. on Turbo Codes \& Iterative Information Processing, Sep. 2010, pp. 196-200.

[6] A. Abrardo, G. Ferrari, and M. Martaló, "On non-cooperative block-faded orthogonal multiple access schemes with correlated sources," Accepted for publication in IEEE Trans. Commun., 2011.

[7] T. Wang, A. Cano, G. B. Giannakis, and J. N. Laneman, "Highperformance cooperative demodulation with decode-and-forward relays," IEEE Trans. Commun., vol. 55, no. 7, pp. 1427-1438, Jul. 2007.

[8] J. Proakis, Digital Communications, 4th ed. McGraw-Hill Science/Engineering/Math, Aug. 2000.

[9] A. Graell i Amat, I. Land, and L. K. Rasmussen, "Error bounds for relaying with decode-and-forward," Submitted to IEEE Trans. Inf. Theory, 2011. 